\title{
AVALIAÇÃO SENSORIAL DA CARNE DE SOL COMERCIALIZADA NAS CIDADES DE CAICÓ E CURRAIS NOVOS
}

\author{
D. S. AMARAL', D. S. G. CARDOSO² T. PESSOA ${ }^{3}$ e F. F. GURJÃO 3 \\ ${ }^{1}$ Instituto Federal do Maranhão - IFMA \\ ${ }^{2}$ Instituto Federal do Rio Grande do Norte - IFRN \\ ${ }^{3}$ Universidade Federal de Campina Grande - UFCG \\ denise.amaral@ifma.edu.br ${ }^{1}$, debycaico@hotmail.com², pessoat@gmail.com³
}

Artigo submetido em abril/2012 e aceito em fevereiro/2014

\section{RESUMO}

A carne de sol é a carne mais conhecida e consumida, e seus sistemas de produção apresentam grande diversidade tecnológica, variando desde os pequenos processamentos em açougues da forma tradicional à industrialização em larga escala, realidade que incide sobre o Padrão de Identidade e Qualidade deste produto. Assim, objetiva-se com este trabalho analisar sensorialmente dois tipos de cortes cárneos mais consumidos nas cidades de Caicó/RN e Currais
Novos/RN, avaliando, através do teste de aceitação, os atributos sabor, aroma, cor, aparência e dureza das carnes de sol Coxão Mole e Alcatra comercializadas nas duas cidades, como também a intenção de compra do consumidor. Portanto, dos quatro tipos de carnes de sol e atributos analisados, a amostra mais aceita também foi a que obteve maior percentual de intenção de compra, sendo o Coxão Mole produzido em Currais Novos/RN.

PALAVRAS-CHAVE: Conservar, Teste de Aceitação, Intenção De Compra.

\section{SENSORY EVALUATION OF SUN-DRIED MEAT SOLD IN THE CITIES OF CAICO AND CURRAIS NOVOS}

\begin{abstract}
The sun-dried meat is the most widely known and consumed meat, and its production systems present a great technological diversity, varying from small butchers processing it in the traditional way to largescale industrialization, a reality that has reflections on the Standard of Identity and Quality of this product. Thus, the objective of this work was to sensory analyze the two most consumed meat cuts in the cities of Caicó/RN and Currais Novos/RN, evaluating, by means of
\end{abstract}

the acceptance test, the flavor, aroma, color, appearance and toughness attributes of the Coxão Mole and Alcatra sun-dried meats commercialized in the two cities, as well as consumer purchase intent. Therefore, of the four types of sun-dried meat and attributes analyzed, the most accepted sample was also the one with highest percentage of purchase intention, being the Coxão Mole produced in Currais Novos/RN.

KEYWORDS: Retaining, Acceptance Testing, Purchase Intention. 


\section{INTRODUÇÃO}

Segundo Feijó (2008), a carne é considerada um alimento nobre para o homem devido produzir energia, novos tecidos orgânicos e a regularização dos processos fisiológicos a partir de gorduras, proteínas e vitaminas. Dentre os principais produtos de carne bovina salgada e dessecada elaborados no Brasil tem-se a carne de sol que, embora bem conhecida no Nordeste brasileiro, no Sul e Sudeste é confundida com o charque e JerkedBeef (Souza, 2005). No processamento desses produtos, inicialmente, as carnes são desossadas, adelgaçadas e cortadas em camadas de 3 a $5 \mathrm{~cm}$ denominadas "mantas" e posteriormente salgadas (Gomez, 2006).

Esta carne é um produto regional que não é produzido em escala industrial e não sofre ação da inspeção veterinária. Desta forma, implica na falta de condições adequadas no que se refere aos aspectos higiênicos- sanitário. A comercialização e distribuição são feitas em feiras livres, mercados municipais, armazéns, supermercados e açougues. O produto é exposto, na maioria das vezes pendurado nos balcões ou disposto em bandejas (Azevedo \& Morais, 2005).

Uma das primeiras tentativas satisfatórias em conservar alimentos cárneos foi por meio de sua exposição, com ou sem salga prévia, aos raios solares. Sendo observado que esse processo mudava as características sensoriais do alimento, tornando-o mais agradável ao paladar humano (FARIAS 2010).Muitos métodos de conservação baseiam-se no controle ou redução do teor de água dos alimentos, procurando associá-los á manutenção da qualidade de uma forma global, mantendo as suas características nutricionais e sensoriais do produto "in natura". Os métodos de secagem, congelamento e adição de soluto são os mais utilizados para esse fim (KIRCHHOF et al., 2008).

Segundo CHIAPPINI (2007) a aceitação dos produtos alimentícios no mercado está na dependência de sua qualidade. O aroma, em particular, está entre os principais atributos que determinam a escolha e o consumo. Historicamente, gregos e romanos perfumavam seus vinhos com rosas, violetas, ervas e condimentos exóticos trazidos da China, Índia e Egito pelos mercadores venezianos.

$\mathrm{Na}$ atualidade, a carne de sol na maioria das capitais nordestinas apresenta índice de aceitação tão expressivo que se pode inferir que seja o alimento predileto dos nordestinos, explica Botelho (2006).

AMARAL et al. (2012) quando estudou o perfil dos consumidores da carne de sol comercializada nos municípios de Caicó e Currais Novos-Rn, percebeu que o almoço é a ocasião de maior consumo e uma freqüência de mais de três vezes por semana, estes apreciam a maciez e preferem a carne magra e seu preparo em fritura na gordura, o consumo expressivo no almoço e a forma de preparo, demonstra que hábitos culturais determinam o comportamento do consumidor de carne de sol.

Por tanto segundo MINIM, LUCIA, e CARNEIRO (2006), tanto as instituições de pesquisa como, principalmente, as indústrias de alimentos, têm buscado identificar e atender os anseios dos consumidores em relação a seus produtos, pois só assim sobreviverão num mercado cada vez mais competitivo. A análise sensorial tem-se mostrado importante ferramenta neste processo, envolvendo um conjunto de técnicas diversas elaboradas com o intuito de avaliar um produto quanto à sua qualidade sensorial, em várias etapas de seu processo de fabricação. É uma 
ciência que objetiva, principalmente estudar as percepções, sensações e reações do consumidor sobre as características dos produtos, incluindo sua aceitação ou rejeição. Diferentes métodos de avaliação são utilizados a fim de se determinar o perfil sensorial, a aceitação, preferências, e intenção de compra acerca de determinados produtos.

Portanto este trabalho tem por objetivo realizar análise sensorial pelo teste de aceitação avaliando os atributos sabor, aroma, cor, aparência, e dureza, como também avaliar a intenção de compra do consumidor em relação as carnes de sol Coxão Mole e Alcatra comercializadas nas cidades de Currais Novos-RN e Caicó-RN.

\section{MATERIAIS E MÉTODOS}

A matéria-prima para esta determinação foi dois tipos de cortes de carne de sol, ou seja, Alcatra e Coxão-mole das cidades de Caicó e Currais Novos no Rio Grande do Norte.

Para realização da análise sensorial a carne foi cortada em forma de cubos, apresentandose tamanhos uniformes, foram pré-cozidas por 30 minutos, para a retirada do excesso de sal,em seguida foram fritas.

A análise foi composta por 40 julgadores não treinados do sexo feminino e masculino, recrutados entre alunos e funcionários do Instituto Federal do Rio Grande do Norte (IFRN) situada na cidade de Currais Novos, Rio Grande do Norte, que receberam esclarecimento de como deviam proceder em suas avaliações.

As amostras foram codificadas com três dígitos escolhidos aleatoriamente, e apresentadas aos provadores juntamente com água, biscoito água e sal e o formulário de avaliação. Estes foram informados a fazer uma pausa entre uma análise e outra, servindo-se desses dois produtos no sentido de minimizar os efeitos do sabor residual deixado na boca pela amostra anteriormente, segundo MININ (2006).

Para a análise sensorial os atributos avaliados foram o sabor, aroma, cor, aparência e dureza, e os julgadores analisaram as amostras pelo teste de aceitação e intenção de compra. Quanto ao teste de aceitação, utilizou-se uma escala estruturada de 1 a 5 em que: 1- Desgostei muito; 2- Desgostei; 3- Não gostei/ Nem desgostei; 4- Gostei e 5- Gostei muito, para a análise de aroma, sabor, cor e aparência. E para a análise de dureza: 1- muito dura; 2- dura; 3- nem dura nem mole; 4- normal e 5- suculenta. Já para o teste de intenção de compra foi utilizada a escala em que: 1- Certamente não compraria; 2- provavelmente não compraria; 3-talvez comprasse/talvez não comprasse; 4- provavelmente compraria; e 5- certamente compraria.

Para a análise dos resultados dos testes sensoriais, utilizou-se o programa ASSISTAT com o qual se realizou a comparação de médias através da ANOVA e, para calcular o grau de concordância entre os consumidores com relação às notas atribuídas utilizou-se o CONSENSOR (SILVA et al., 2010). 


\section{RESULTADOS E DISCUSSÕES}

\subsection{Teste de Aceitação}

Na Tabela 1 estão às médias das notas atribuídas pelos provadores e coeficiente de concordância para os atributos sensoriais (sabor, aroma, aparência e cor) dos diferentes tipos de carne de sol.

Tabela 1- Média das notas atribuídas pelos provadores e coeficiente de concordância para os atributos sensoriais (sabor, aroma, aparência e cor) dos diferentes tipos de carne de sol: AL CA (Alcatra Caicó), AL CU (Alcatra Currais Novos), CM CA (Coxão Mole Caicó), CM CU (Coxão Mole Currais Novos).

\begin{tabular}{|l|c|c|c|c|c|c|c|c|c|c|}
\hline \multirow{2}{*}{$\begin{array}{c}\text { Carne } \\
\text { de sol }\end{array}$} & \multicolumn{2}{|c|}{ Sabor } & \multicolumn{2}{c|}{ Aroma } & \multicolumn{2}{c|}{ Cor } & \multicolumn{2}{c|}{ Aparência } & \multicolumn{2}{c|}{ Dureza } \\
\cline { 2 - 13 } & Média & $\mathrm{CC}(\%)$ & Média & $\mathrm{CC}(\%)$ & Média & $\mathrm{CC}(\%)$ & Média & $\mathrm{CC}(\%)$ & Média & $\mathrm{CC}(\%)$ \\
\hline AL CA & 3,4 & 42,17 & 3,5 & 40,82 & 3,67 & 33,33 & 3,23 & 41,16 & 3,43 & 22,98 \\
\hline AL CU & 3,3 & 44,41 & 3,53 & 41,16 & 3,17 & 19,41 & 3,1 & 28,34 & 3,5 & 46,25 \\
\hline CM CA & 2,47 & 23,57 & 3,17 & 34,56 & 2,77 & 15,67 & 3 & 14,9 & 2,17 & 32,05 \\
\hline CM CU & 3,83 & 41,83 & 3,27 & 11,79 & 3,67 & 27,39 & 3,73 & 35,75 & 3,77 & 31,06 \\
\hline
\end{tabular}

O Coeficiente de concordância (CC\%) calcula o grau de concordância entre os provadores, com relação à aceitação ou reprovação das amostras apresentadas.

Na Tabela 1, percebe-se que os maiores coeficientes foi dado para o atributo Sabor e Dureza para a carne de sol produzida na cidade de Currais Novos-RN com o corte Alcatra, sendo 44,41 e 46,25\%, respectivamente. Portanto dos 40 provadores, mais de 18 concordam entre si com a média acima de 3,0 (não gostei/ nem desgostei), e próximo a 4 (gostei).

Verifica-se que para os dois tipos de corte das cidades analisadas, a Alcatra produzida em Caicó-RN, apresentou os maiores valores do coeficiente de concordância, sendo estes acima de $23 \%$, isto demostra que ocorreu uma maior concordância entre as respostas dos 40 provadores.

Na Tabela 2 tem-se o teste de médias da análise sensorial para os diferentes tipos de carne de sol: AL CA (Alcatra Caicó), AL CU (Alcatra Currais Novos), CM CA (Coxão Mole Caicó), CM CU (Coxão Mole Currais Novos).

Tabela 2- Teste de médias da análise sensorial para os diferentes tipos de carne de sol: AL CA (Alcatra Caicó), AL CU (Alcatra Currais Novos), CM CA (Coxão Mole Caicó), CM CU (Coxão Mole Currais Novos).

\begin{tabular}{|c|c|c|c|c|c|}
\hline \multirow{2}{*}{$\begin{array}{c}\text { Carne } \\
\text { de sol }\end{array}$} & \multicolumn{5}{|c|}{ Média } \\
\cline { 2 - 6 } & Sabor & Aroma & Cor & Aparência & Dureza \\
\hline AL CA & $3,40 a$ & $3,50 a$ & $3,67 a$ & $3,23 a$ & $3,43 a$ \\
\hline AL CU & $3,30 a$ & $3,53 a$ & $3,17 a b$ & $3,10 a$ & $3,50 a$ \\
\hline CM CA & 2,46 b & $3,17 a$ & 2,77 b & $3,00 a$ & $2,17 b$ \\
\hline CM CU & $3,83 a$ & $3,27 a$ & $3,67 a$ & $3,73 a$ & $3,77 a$ \\
\hline C.V\% & 30,38 & 31,80 & 35,50 & 33,62 & 32,96 \\
\hline
\end{tabular}

As médias seguidas pela mesma letra não diferem estatisticamente entre si, pelo teste de Tukey ao nível de $5 \%$ de probabilidade.

$\mathrm{Na}$ Tabela 2, pode-se observar, quanto ao atributo Aroma e Aparência, que as médias atribuídas pelos provadores que analisaram os quatro tipos de carnes não diferiram estatisticamente entre si, ou seja, são iguais quando testadas pelo teste Tukeyao nível de $5 \%$ de probabilidade, estas médias apresentaram-se superior a 3 e inferior a 4, representando assim dúvida em relação ao gosto ou desgosto desse atributo. 
Para o atributo Sabor e Dureza as carnes Alcatra de Caicó-RN e de Currais Novos-RN e o Coxão Mole de Currais Novos-RN apresentaram-se estatisticamente iguais, com médias acima de 3,40 e diferiram-se somente do Coxão Mole produzido em Caicó-RN, o qual apresentou médias abaixo de 2,46 e 2,17, sendo este valor correspondente a Desgotei.

No atributo Cor, nota-se que a Alcatra de Caicó-RN, a de Currais Novos-RN e o Coxão Mole de Currais Novos-RN são estatisticamente iguais, assim como a Alcatra de Currais Novos-RN e o Coxão Mole de Caicó-RN. Verifica-se em todos os atributos que o coeficiente de variação (C.V) apresentou-se elevado, pois trata-se de 40 provadores distintos não treinados.

Tem-se na Figura 2 a comparação do perfil sensorial para os diferentes tipos de carne de sol: AL CA (Alcatra Caicó), AL CU (Alcatra Currais Novos), CM CA (Coxão Mole Caicó), CM CU (Coxão Mole Currais Novos).

Com o objetivo de facilitar a visualização da comparação das médias dos tipos de cortes das duas cidades, construíu-se a Figura 2. Nesta os pontos mais afastados do centro representam a amostra de maior preferência pelos consumidores e quanto mais próxima ao centro menos preferida foi a amostra, sendo assim a amostra que apresentou os maiores valores de media para os atributos foi o Coxão Mole produzido em Currais Novos-RN, seguido da Alcatra de CaicóRN que quase não diferiu da de Currais Novos-RN, porém a menos aceita foi o Coxão Mole de Caicó-RN.

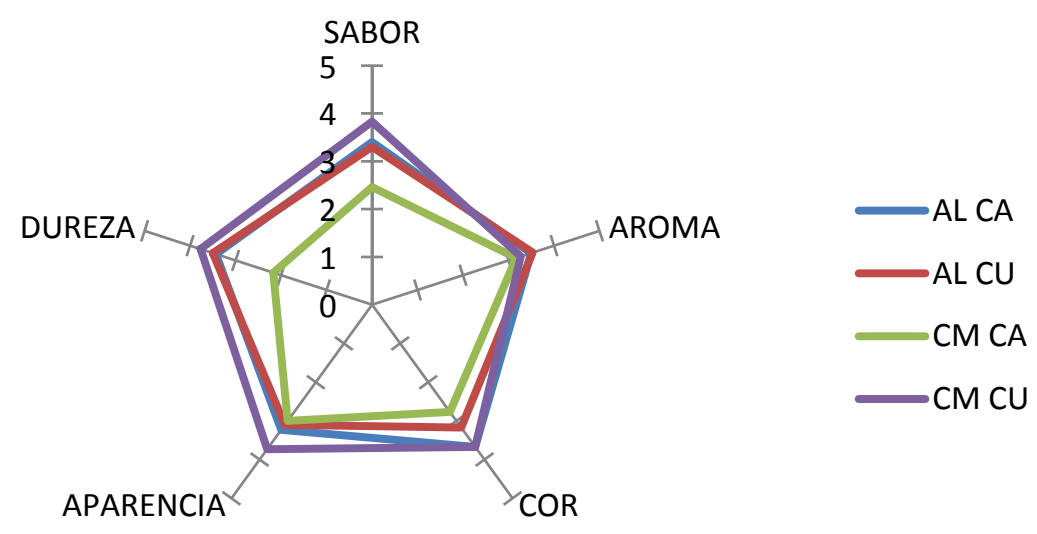

Figura 2- Comparação do perfil sensorial para os diferentes tipos de carne de sol: AL CA (Alcatra Caicó), AL CU (Alcatra Currais Novos), CM CA (Coxão Mole Caicó), CM CU (Coxão Mole Currais Novos).

\subsection{Intenção de Compra}

Na Figura 3 tem-se o percentual de intenção de compra para os diferentes tipos de carne de sol: AL CA (Alcatra Caicó), AL CU (Alcatra Currais Novos), CM CA (Coxão Mole Caicó), CM CU (Coxão Mole Currais Novos). Nesta, nota-se que os maiores percentuais de intenção de compra foram atribuídos a Provavelmente e Certamente Comprariam, o que demonstra uma boa aceitação das amostras, mas os maiores percentuais dados pelos provadores para Certamente Compraria foi para o Coxão Mole de Currais Novos-RN e Alcatra de Caicó-RN, 37 e 30\%, respectivamente. 


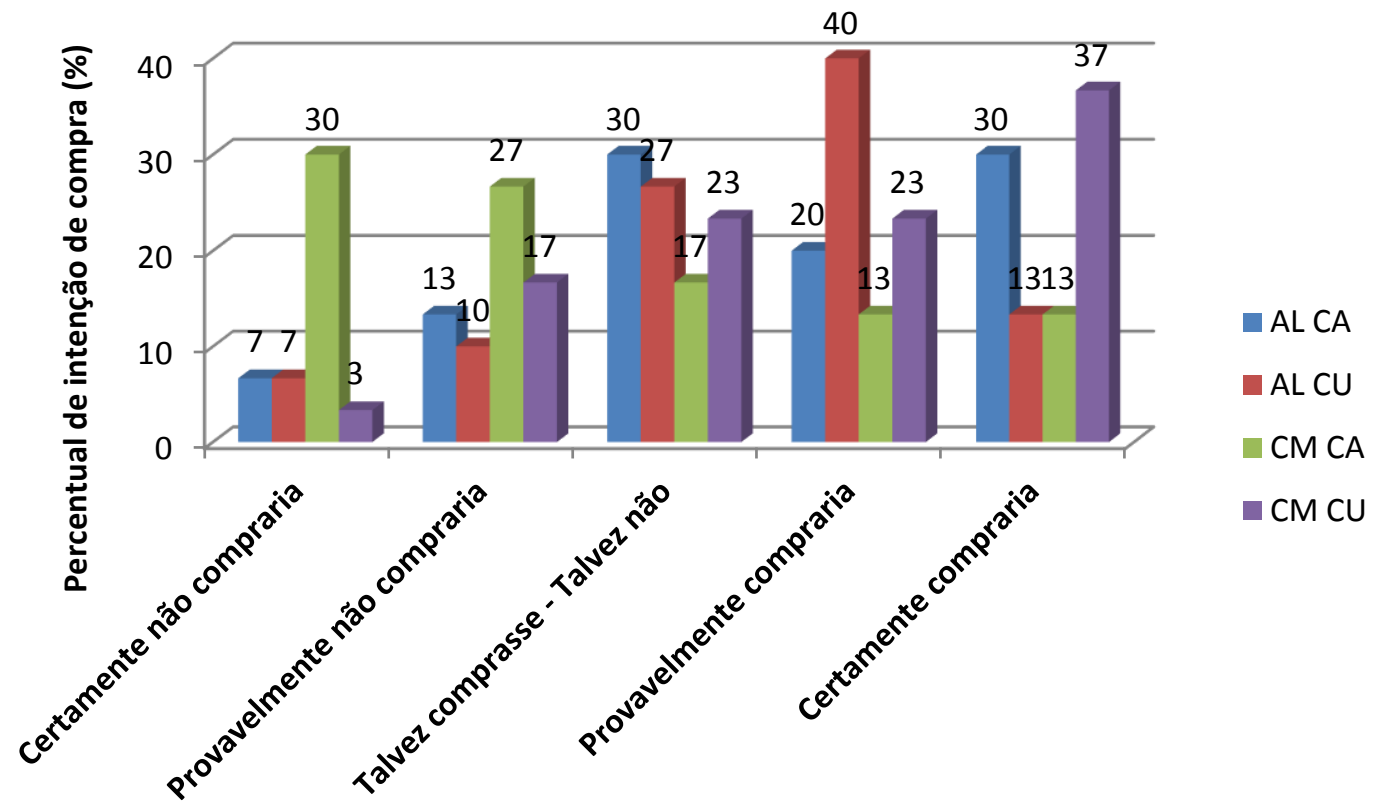

Figura 3-Percentual de intenção de compra para os diferentes tipos de carne de sol: AL CA (Alcatra Caicó), AL CU (Alcatra Currais Novos), CM CA (Coxão Mole Caicó), CM CU (Coxão Mole Currais Novos).

\section{CONCLUSÕES}

Os maiores valores de CC para todos os atributos foram para a Alcatra de Caicó-RN, e os maiores percentuais de intenção de compra foram atribuídos a Certamente Compraria o Coxão Mole de Currais Novos-RN e a Alcatra de Caicó-RN.

Para os quatro tipos de carnes de sol e os atributos analisados, a amostra mais aceita foi a que obteve maior percentual de intenção de compra, sendo o Coxão Mole produzido em Currais Novos-RN, apresentando-se médias de aceitação próximas a 4 (gostei) e $37 \%$ percentual de intenção de compra.

\section{AGRADECIMENTOS}

Ao IFRN de Currais novos pela disposição do espaço para realização desta pesquisa.

\section{REFERÊNCIAS BIBLIOGRÁFICAS}

1. AMARAL, D. S.; CARDOSO, D. S. G. PESSOA, T.; NETO, L. G. M. Perfil dos consumidores da carne de sol comercializada nos municípios de Caicó e Currais Novos. Acta veterinária Brasilica, V.6, n.4 p. 302- 211, 2012.

2. Azevedo, A. R. P. \& Morais, T. V. M. A tecnologia da produção da carne-de-sol e suas implicações nos aspectos higiênicos-sanitários. Rev. Nacional da Carne. 336: 36-50, 2005.

3. Botelho, R. B. A. Cultura Alimentar e Alimentação Saudável. Tese de doutorado, Faculdade de Ciências da Saúde da Universidade de Brasília, Brasília, 192p, 2006.

4. CHIAPPINI, C. C de J. Aromas naturais produzidos por microorganismos. Revista Eletrônica de Jornalismo Científico, set, 2007. Disponível em: http://www.comciencia.br/comciencia/ 
?section=8\&edicao=28\&id=325. Acesso em 25 de out. 2011.

5. FARIAS, S.M.O.C.; Qualidade da carne de sol comercializada na Cidade de João Pessoa. 2010. 142f. Dissertação (mestrado)- Curso de ciência e tecnologia de alimentos da Universidade Federal da Paraíba- UFPB, João Pessoa, 2010.

6. Feijó, G. P. I. Curso "Conhecendo a carne que você consome". Composição química da carne bovina. Gado de Corte. Capturado em 25 de out. de 2011. Online. Disponível em: http://www.cnpgc.embrapa.br/publicacoes/doc/doc77/03nocoescarne.html\#3.2.

7. Gomez. JerkedBeef fermentado: desenvolvimento de nova tecnologia de processamento. Dissertação de mestrado, Universidade Estadual de Londrina, Londrina, 114p, 2006.

8. LUCIA, S.M.D., MINIM,V.P.R., CARNEIRO, J.D.S. Análise Sensorial de Alimentos. In:MINIM, V.P.R. Análise Sensorial Estudos com Consumidores. Viçosa: UFV, 2006. 13-49.

9. KIRCHHOF, S.; CRIZEL, G. R.; MENDONÇA, C. R. B. In:VII CONGRESSO DE INICIAÇÃO CIENTífICA, 2008, Pelotas, A influência da água na conservação dos alimentos. Pelotas: UFPEL, 2008.

10. SILVA, F. A. S.; DUARTE, M. E. M.; CAVALCANTI MATA, M. E. R. M. Nova metodologia para interpretação de dados de análise sensorial de alimentos. Revista Engenharia Agrícola.v. 30, n.5, p.967-973, set./out. 2010.

11. Souza, N. L. 2005. Efeito da combinação de sal com lacto e diacetato de sódio nas características sensoriais, físico-químicas, cor e textura de um produto similar à carne-de-sol. Dissertação de mestrado, Universidade Estadual de Campinas, Campinas. 